\title{
An overview of ground-penetrating radar signal processing techniques for road inspections
}

\author{
Andrea BENEDETTO ${ }^{1}$, Fabio TOSTI ${ }^{2, *}$, Luca BIANCHINI CIAMPOLI ${ }^{1}$, Fabrizio D’AMICO ${ }^{1}$
}

${ }^{1}$ Department of Engineering, Roma Tre University, Via Vito Volterra 62, 00146, Rome, Italy; e-mail: andrea.benedetto@uniroma3.it; luca.bianchiniciampoli@uniroma3.it; fabrizio.damico@uniroma3.it

${ }^{2}$ School of Computing and Engineering, University of West London (UWL), St Mary's Road, Ealing, London W5 5RF; Fabio.Tosti@uwl.ac.uk (*Corresponding author)

\begin{abstract}
Ground-penetrating radar (GPR) was firstly used in traffic infrastructure surveys during the first half of the Seventies for testing in tunnel applications. From that time onwards, such non-destructive testing (NDT) technique has found exactly in the field of road engineering one of the application areas of major interest for its capability in performing accurate continuous profiles of pavement layers and detecting major causes of structural failure at traffic speed. This work provides an overview on the main signal processing techniques employed in road engineering, and theoretical insights and instructions on the proper use of the processing in relation to the quality of the data acquired and the purposes of the surveys.
\end{abstract}

Keywords: ground-penetrating radar; GPR; pavement engineering; data processing for road inspections

\section{INTRODUCTION}

Safety concerns and economical savings in road construction and maintenance strategies are no doubt the two main issues around which considerable efforts of engineers and practitioners are being increasingly focused. In terms of driving safety, it is well known how cracks, potholes, and surface deformations can generate sudden vertical accelerations on the vehicle tires, thereby lowering the effective friction between tires and pavement surface and raising the probability of car accidents [1]. As far as the economic aspect is concerned, three main factors have a great influence nowadays in orienting the policies of investment of governments and local authorities in the transportation area, namely, i) the general lack of economic resources which causes a lowering of the demand for new constructions; ii) the need for a road asset that can 
meet the current requirements of mobility, and iii) the progressive aging of existing assets. Accordingly, effective road maintenance policies can be considered nowadays as important as good construction practices. Within the frame of road inspection methods, traditional techniques such as coring and drilling are based on a destructive approach. Notwithstanding the high reliability, they reveal as expensive, time-consuming and slightly significant, since the information gathered can not be extended to long-range distances. Thereby, several non-destructive testing or evaluation (NDT/NDE) techniques have been developed to enable more efficient assessments of road pavements and materials. Among the major advantages, we can mention the faster data acquisition, more contained cost, and the capability to be performed in-situ over longer distances $[2,3]$.

Within the various NDTs employed in road surveys, ground-penetrating radar (GPR) is nowadays established as one of the most effective and powerful, due to its high flexibility and reliability of results. Basically, this tool enables to infer information on the physical and geometrical conditions of the subsurface relying on the transmission and reception of short EM impulses in a given frequency band [4, 5].

First documented research on the use of GPR in the traffic infrastructure area can be traced back in the 1970s by the Federal Highway Administration (FHWA) for testing in tunnel investigation [6]. The main applications in this area concern the inspections of pavement layers, and they broadly include the evaluation of layer thicknesses [7], the assessment of damage conditions in hot mix asphalt (HMA) layers [8], loadbearing layers and subgrade soils [9], and the inspection of concrete structures [10, 11]. New frontiers on the possibility to infer strength and deformation properties of road pavements and materials from their EM characteristics have been also recently explored $[12,13]$. In addition, finite-difference time-domain (FDTD) simulation-based approaches of the GPR signal have been implemented for analysing the EM response of typical scenarios of pavement faults [14].

The aforementioned applications require suitable processing schemes to GPR data for providing easily interpretable images to operators and decision-makers [4]. Indeed, one of the final goals of the signal processing is to improve the quality of the data collected, which is mainly related to an increase of the signalto-noise ratio (SNR). Nevertheless, the risk of achieving distorted information by over-processing the data is considerable, and it tends to rise the more sophisticated is the processing technique applied. 
Overall, it can not be universally recognized an established and unique processing scheme to follow. The best way to treat the collected data is highly dependent on several factors. Firstly, a suitable stage of data collection on the field is the most important step to undertake for a later and successful interpretation of data, regardless of the quality and depth of the applied processing. Thereby, an aware and strict survey protocol [15] will imply, most likely, a faster and smoother phase of processing. Secondly, the rate of complexity in getting the aim of the survey heavily affects the processing procedures. The more complex is the information to retrieve, e.g., detection of buried targets in strongly anthropic environments, the higher is the processing effort required. Finally, it is worth mentioning the costs related to the data processing, especially in terms of human resources and time invested. Accordingly, it is crucial to evaluate comprehensively the objectives of a GPR survey and the deliverables of each processing technique fits for purposes [15].

As far as the road applications are concerned, the multi-layered horizontal character of pavements along with a relatively widespread knowledge a-priori of the construction materials of each layer, tends to lower the risk of over-processing the data. Therefore, the applicability of specific and more advanced processing schemes can increase significantly the quality of the data interpretation $[4,16]$. On the other hand, wrong processing applications are likely to increase due to the typical large amount of data gathered in road surveys, and aware and proper post-processing phases are crucial to be planned.

This work presents an overview on the main signal processing techniques employed in road engineering using GPR. Section II deals with the theoretical bases of GPR useful to frame the context in which the signal processing is performed. Subsequently, the main processing techniques are discussed in Section III, wherein the main benefits achieved in a number of significant GPR applications in road engineering are highlighted. Finally, conclusions and future perspectives are drawn in Section IV.

\section{GPR PRINCIPLES AND MAIN CONFIGURATIONS}

\subsection{The GPR technique}

GPR is a geophysical inspection technique that operates by transmitting electromagnetic (EM) waves toward a surface, typically a soil, and by receiving the transmitted or back-reflected signal. The propagation of the EM waves is ruled by the dielectric properties of the medium passed through, namely, the dielectric 
permittivity $\varepsilon$, the electric conductivity $\sigma$ and the magnetic permeability $\mu$. In particular, $\varepsilon$ and $\sigma$ greatly influence the behavior of the propagating wave, in terms of wave velocity and wave attenuation, respectively, and $\mu$ is equal for all the non-magnetic materials to the free space magnetic permeability $\mu_{0}$ and does not affect the propagation of the EM wave. Practically, the dielectric contrasts in the medium generate a partial reflection and transmission of the EM impulse emitted by a transmitting source. Depending on the operating mode, the reflected or transmitted part of the signal is collected by a receiving antenna, and allows imaging the subsurface, in both two or three dimensions. Penetration depth and spatial resolution are influenced by several factors, amongst which the frequency of the emitted signal and the type of material investigated are worth to be mentioned. In civil engineering applications, GPR works typically in a range of frequency between $100 \mathrm{MHz}$ and $2000 \mathrm{MHz}$ [17].

Theoretically speaking, the physics of EM fields is described by the Maxwell's equations, as follows:

$$
\begin{aligned}
& \nabla \times \vec{E}=-\frac{\partial(\vec{B})}{\partial t} \\
& \nabla \times \vec{H}=\vec{J}+\frac{\partial(\vec{D})}{\partial t} \\
& \nabla \cdot \vec{D}=q \\
& \nabla \cdot \vec{B}=0
\end{aligned}
$$

with $\vec{E}\left(\mathrm{~V} \cdot \mathrm{m}^{-1}\right)$ being the strength vector of the electric field, $q\left(\mathrm{C} \cdot \mathrm{m}^{-3}\right)$ the electric charge density, $\vec{B}(T)$ the density vector of the magnetic flux, $\vec{J}\left(\mathrm{~A} \cdot \mathrm{m}^{-2}\right)$ the density vector of the electric current, $\vec{D}\left(\mathrm{C} \cdot \mathrm{m}^{-2}\right)$ the electric displacement vector, $t$ (s) the time, and with $\vec{H}\left(\mathrm{~A} \cdot \mathrm{m}^{-1}\right)$ being the intensity vector of the magnetic field.

Conversely, the behavior of the medium wherein the EM wave is propagating, can be described by the constitutive relationships, reported as follows:

$$
\vec{J}=\sigma \vec{E}
$$




$$
\begin{aligned}
& \vec{D}=\varepsilon \vec{E} \\
& \vec{B}=\mu \vec{H}
\end{aligned}
$$

By combining the EM fields' theory with the material properties, it is possible to describe comprehensively the GPR signals.

\subsection{Main GPR configurations}

The application of GPR in road engineering is mostly related to the use of impulse radar systems, due to a major easiness of usage and data interpretation. These systems operate by transmitting toward the target a very short pulse $\left(\sim 10^{-9} \mathrm{~s}\right)$, characterised by a fixed central frequency, by means of one or more antennas, and by recording the signal back-reflected by the dielectric discontinuities. The two-way travel time signal is then recorded in the time domain, and a map of reflections generated in the subsurface can be finally displayed.

Conversely, stepped frequency continuous-wave (SFCW) radar systems, operate in the frequency domain. Amplitude and phase of the emitted and received signal are sampled and collected as a function of the frequency, which is linearly incremented of a fixed step within a defined frequency range. The high costs of electronics, a major complexity of data processing and the lack of dedicated commercial software have broadly resulted in a lower spread of SFWC radars in road engineering, although their use is recently increased [18].

Concerning the configurations of the antennas, a GPR system is configured as mono-static when a unique antenna operates as both transmitter and receiver. Conversely, in case of separated transmitter and receiver, the GPR system is defined as bi-static. Furthermore, GPR systems can be sorted in ground-coupled and aircoupled according to the type of antenna. In the first case, the antenna is in direct contact with the ground. This allows higher depths of penetration throughout the medium. Central frequencies usually available for this configuration range from $80 \mathrm{MHz}$ to $1500 \mathrm{MHz}$. In the second survey configuration, the antenna is typically kept at a constant height above the surface, mostly ranging from $0.15 \mathrm{~m}$ to $0.50 \mathrm{~m}$. Most common air-coupled GPR systems are pulsed systems operating in the range $0.5 \div 2.5 \mathrm{GHz}$, with a central frequency typically of $1 \mathrm{GHz}$. The penetration depth of an air-coupled system is dependent on the central frequency and 
rarely exceeds $0.9 \mathrm{~m}$ for pavement applications [19]. Despite this main drawback, air-coupled systems allow to survey at traffic speed by mounting the system onto an instrumented vehicle [20]. The advantage of avoiding traffic interruption makes the air-couple GPR systems the most common devices used in road surveys.

\subsection{Radar signal imaging}

There are a multitude of ways for displaying a GPR response, as shown in Fig. 1. In general, it is possible to represent the signal collected as:

$$
f(x, y, z)=A\left(x_{i}, y_{j}, z_{k}\right)
$$

with $i, j$ and $k$ ranging from 1 to $N, M, P$, respectively.
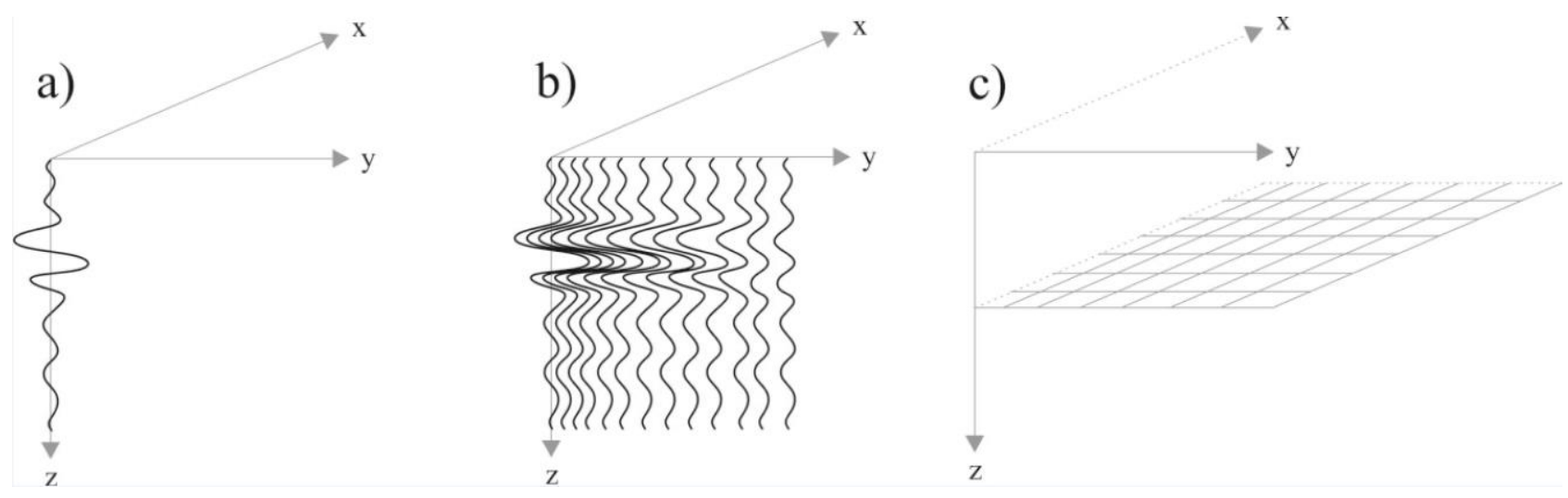

Fig. 1. Visualization modes of a GPR signal: (a) A-scan, (b) B-scan, and (c) C-scan.

A single radar trace, or waveform, is called A-scan (Fig. 1a), and it can be defined as:

$$
f(z)=A\left(x_{i}, y_{j}, z_{k}\right)
$$

with $k$ ranging from 1 to $P, i$ and $j$ equal to a constant value. The A-scan provides a punctual information about the subsurface configuration. It is worth to note how the $z$-axis can represent both time and depth, which are related each other by the propagation velocity.

A set of consecutive radar waveforms along a particular direction ( $x$, for instance) represents a B-scan (Fig. 1b), and it can be defined as follows:

$$
f(x, z)=A\left(x_{i}, y_{j}, z_{k}\right)
$$


with $i$ and $k$ ranging from 1 to $N$ and $P$, respectively, and $j$ being a constant value. Therefore, a B-scan visualization mode allows to achieve a two dimensional view along a specific direction. In road engineering applications, the B-scan mode is a widely used imaging methodology, wherewith the variations occurring on the main geometrical features of the pavement, such as the thickness of the layers, can be relatively easily detected.

If a value along the $z$-axis is fixed as constant, the visualization of the horizontal domain $(x, y)$ is called $\mathrm{C}$ scan, and it is defined as follows:

$$
f(x, y, z)=A\left(x_{i}, y_{j}, z_{k}\right)
$$

over the range $i=1$ to $N, j=1$ to $M$ and $k=$ constant. Practically, the C-scan view mode provides an amplitude map at a specific time of collection. When one area is surveyed, for instance by collecting data along a regular grid, the C-scan visualization mode can be very useful in detecting inhomogeneous spots, which are characterized by a high reflectivity in terms of signal amplitudes.

Overall, the GPR data are processed and depicted according to the above three visualization modes. Since the A-scan mode provides information on the temporal delay between the emission and the reception of a single transmitted or back-reflected signal, the processing of the single A-scan operates exclusively in the punctual spatial domain influencing that signal. When a set of consecutive waveforms is considered, such temporal frames are gradually set beside along a linear track. Therefore, the processing of a B-scan operates in both temporal (i.e., in depth along the $z$ axis) and spatial (i.e., along the path of the scan, namely, the $x$ axis) dimensions. The C-scan visualization concerns a defined temporal instant, such that the processing operates only in space. Due to the considerable extension of roads and the resultant huge amount of data processing required, the $\mathrm{C}$-scan visualization mode is rarely used in road applications. Accordingly, the processing related to the $\mathrm{C}$-scan visualization will not be tackled in this study.

\section{SIGNAL PROCESSING TECHNIQUES IN ROAD INSPECTIONS}

Signal processing in the GPR area exploits many of the advances achieved in the early eighties in the digital processing of seismic data [21,22]. Although these two disciplines hold evident differences in the source of propagation, they share identical basics under a processing perspective, which consists in a collection of 
pulsed signals in the time domain. The main difference between such two areas lies in the nature of the emitted and received wave, which in seismic is mechanical, whereas in GPR is EM-based. EM waves are defined as 'non-stationary', since they show a faster decay of the amplitude with the penetration through the soil, and the loss of higher frequency harmonics [19]. Besides, GPR waves suffer higher scattering phenomena, ruled by complex reflection coefficients and major similarity between the wavelength and the discontinuities in the media than in seismic. Nevertheless, many of the processing techniques performed when tackling a GPR dataset originate from the seismic theory.

In this Section, the main processing techniques for GPR dataset in road surveys are presented. The discussion will be sorted by i) processing techniques required to be performed prior to any post-processing step, ii) techniques involving the processing of A-scan data, and iii) those dealing with B-scan data.

\subsection{Basic processing}

\subsubsection{Data editing}

This step represents the first preliminary activity to be carried out prior to approach any data processing. Mostly, the data collected need to be sorted and arranged to secure highly-reliable interpretation, especially in the case of considerable amounts of data, such as in road investigations.

A first level of faults in the data is generally related to incorrect or inaccurate settings of the survey parameters.

A second level is rather related to faults occurring during the collection. The quality of the visualization of a particular survey section can be affected significantly by incoherent, fuzzy or clipped traces. This contingency is not rare and tends to reduce as the expertise of the surveyor increases. The main causes of misstated traces are the external sources of noise and the failures of the GPR equipment. For example, excessive travel speeds can cause such type of issues, since the probability of asynchrony between the GPR trace and the odometer tends to raise.

In the case of singular traces or a subtle band of traces, a simple cut of the corrupted traces and an interpolation between previous and following traces is usually sufficient. When errors get more frequent, repeated interpolations are necessary, and the risk of losing information increases. 
A particular error occurring in GPR acquisitions is the clipping of the initial ground wave signal, i.e., when a strong air-pavement coupling saturates the GPR receiver, as shown in Fig. 2a. This is more common in case of ground-coupled antennas. When clipping occurs, the collected signal is not representative of the real peak amplitude reflected at the ground surface. Moreover, if a normalization to the peak amplitude value is performed, an artificial enhancement of the late-arrival reflections for the saturated traces in respect to the non-saturated ones will be recorded. In such a case, the desaturation function is a useful tool for reconstructing the true shape of the amplitude peak by using a spline interpolation (Fig. 2b).
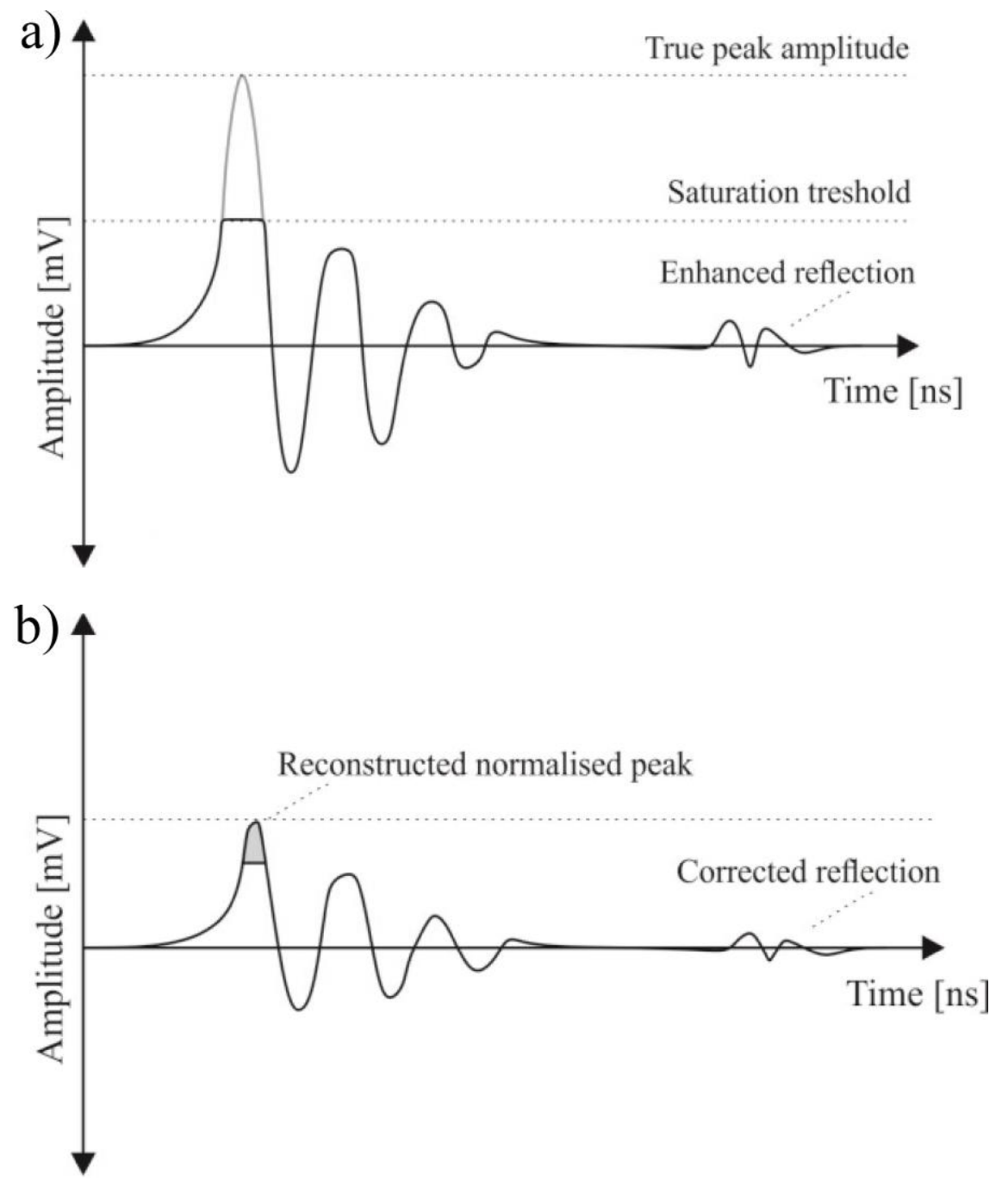

Fig. 2. Clipping errors of a GPR signal: (a) clipping of the initial ground wave signal, and (b) reconstructed signal. 
When no odometer is employed, the pulse is emitted by the GPR system at regular time intervals. This can represent a critical issue, in case more advanced processing procedures are subsequently implemented. Although the surveying speed is maintained as constant as possible, a regular collection of radar traces in spatial terms is indeed impossible to perform [23]. In such a case, the GPR sections appear stretched or compressed. This issue can be sorted out by marking the radar collection with a regular step, e.g., $100 \mathrm{~m}$, and by resampling the data in order to generate sections with equally-spaced traces, namely, by performing a "rubber-band interpolation".

\subsubsection{Time-zero correction}

In road surveys it is strictly necessary to have a fixed and unique reference as a time-zero point for the GPR data, in order to compare the reflection time and, possibly, the depth of inhomogeneities located at different positions along the survey track. Mostly, this can not be ensured due to several factors, such as the different temperature of the air during the collection of the data, the different length of the connecting cables or, more simply, the variation of the antenna height due to the vertical acceleration acting on the instrumented vehicle $[23,24]$. Therefore, the position of the reflection coming from the air-pavement interface can vary between different A-scans (Fig 3a). To avoid the obvious interpretation issues arising from a variable time-zero reference, and to allow the employment of further processing techniques, the data need a correction to set a common time-zero position (Fig 3b). Mainly, this issue is sorted by cutting the air layer to a fixed threshold, set at a mostly stable point of the considered trace. Depending on both the type of the antenna and the central frequency of investigation, setting the proper position of this threshold along the A-scan reflects on the accuracy of the results. According to [25], among the possible thresholds, the most employed by users and advised by manufacturers can be summarized as i) the first break-point, ii) the first negative peak, iii) the zero-amplitude point between the negative and the positive peaks, iv) the mid-amplitude point between the negative and the positive peaks and v) the first negative peak. Each method holds advantages and drawbacks with regard to the dieletric properties of the surface materials and the central frequency of investigation [25]. Nevertheless, an on-site calibration of the time-zero is always required, and the picking of any features within the A-scans with the evaluation of the relevant depths must account every time for the chosen method. 

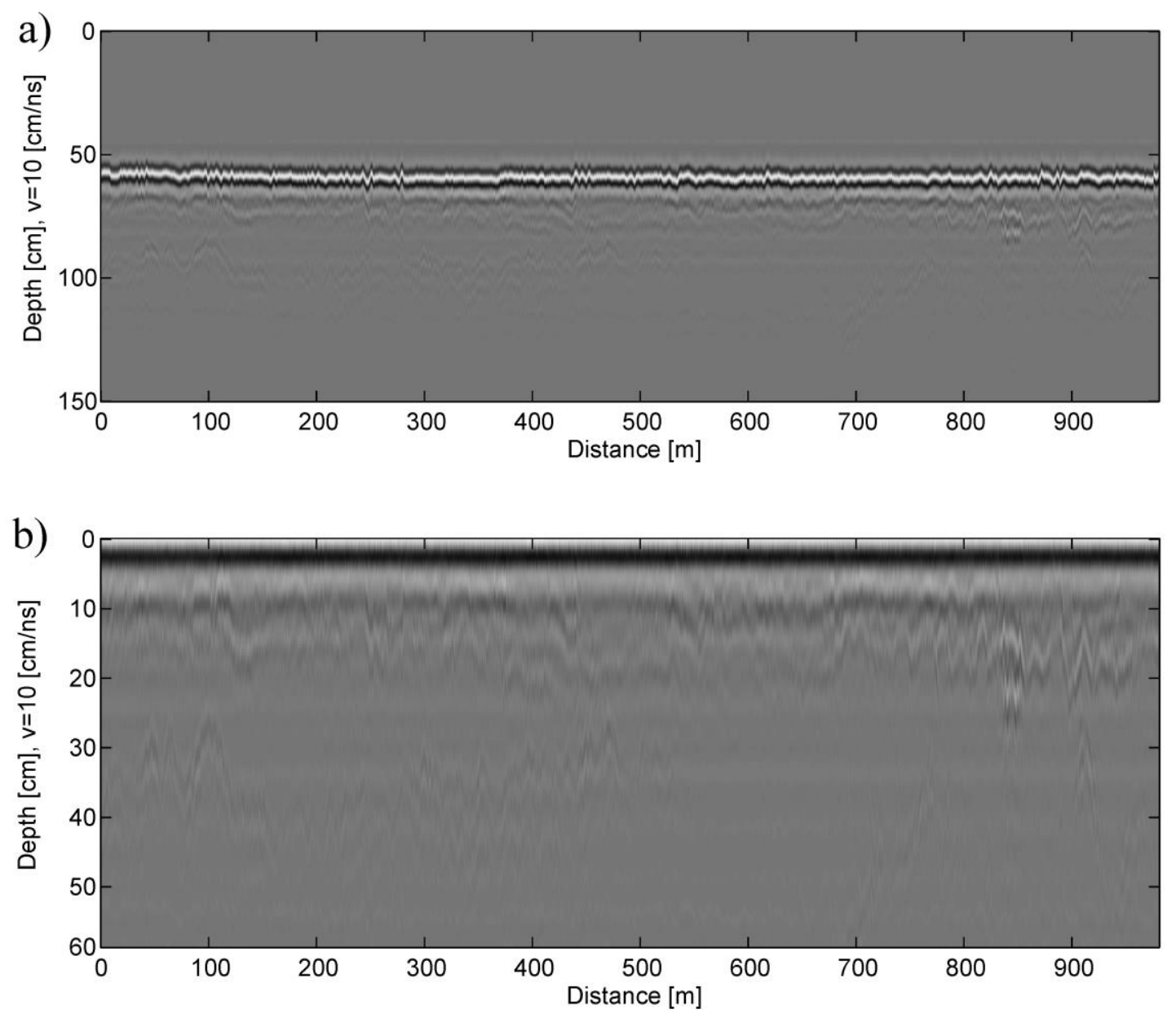

Fig. 3. B-scan representation of a road survey: (a) raw B-scan before time-zero correction and, (b) processed B-scan after time-zero correction using a common time-zero position.

\subsection{A-scan processing}

\subsubsection{Zero offset removal}

The initial direct current (DC) signal component and the very low-frequency signal trend (or 'wow') can generate a distortion of the mean of the A-scan towards values of amplitude far from zero [26]. This occurrence is partially related to the coupling effect and to the saturation of the signal by early arrivals, and itis not usually negligible in road inspections, since a strong dielectric contrast between the air and the surface of the pavement typically occurs. This affects the spectrum of the trace and inhibits further spectral processing steps or time varying gains [27]. Mostly, processing software are capable to sort out this problem by using simple average-subtraction algorithms, such as the following:

$$
y^{\prime}(n)=y(n)-\frac{1}{N} \sum_{k=1}^{N} y(k)
$$


with $y(n)$ and $y_{r}(n)$ being the amplitude of the $n^{\text {th }}$ sample of the processed and raw trace, respectively, and with $n$ ranging from 1 to $N$. The result of the application of this algorithm turns out to be an A-scan with mean equal to zero, which means a symmetric probability distribution of the amplitude along the A-scan (see Fig. 4).

The same goal can be granted by applying a high-pass filter and removing the low-frequency signal components. More details about this method are given in Section 3.2.2.

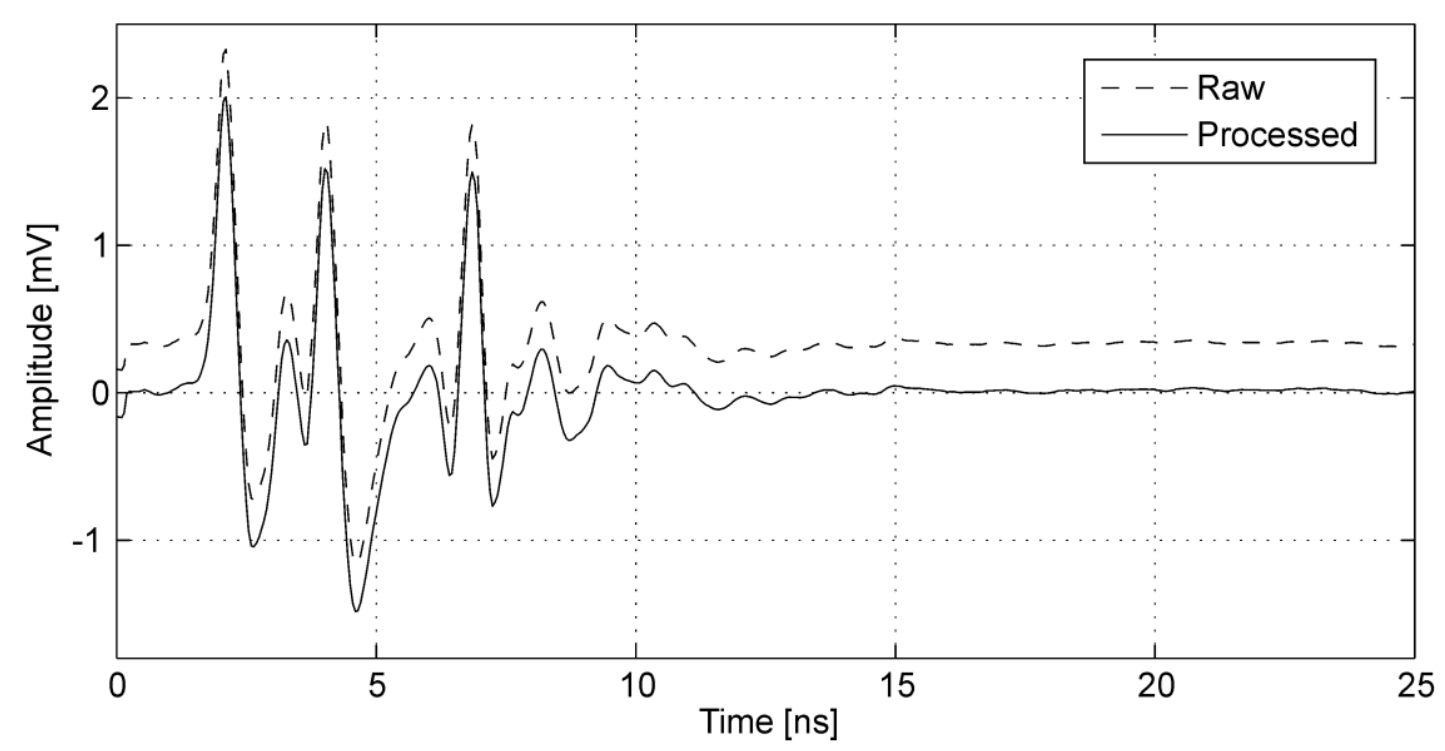

Fig. 4. Zero offset removal in a typical GPR trace of a road section.

\subsubsection{Band-pass filtering}

The application of a band-pass filter may represent a crucial step for a correct visualization and interpretation of a GPR signal [28-30]. This processing method is aimed at increasing the SNR by filtering out from the data the signal components with frequencies outside the main working bandwidth of the GPR system employed.

A band-pass filter can be considered as the combination of two frequency filters, namely, the high-pass and the low-pass filters. The first one operates a cut-off of the low frequency components from the frequency 
spectrum of each singular trace. This allows to filter the clutter related to both the ground-wave and the 'cultural sources' of noise, such as nearby vehicles, buildings, fences, power lines or trees in close proximity to the roadway [31-33]. The low-pass filter works by cutting off the high frequency components from the spectrum, which are usually generated by the EM interferences between the antenna and relevant everyday EM devices, such as mobile phones.

Fig 5 shows the spectral visualization of a GPR signal, highly affected by both low and high frequency clutter, collected in a road inspection with an air-launched GPR system equipped with a $1 \mathrm{GHz}$ central frequency horn antenna. In particular, it is possible to recognize a significant component of the signal affecting frequencies below $~ 350 \mathrm{MHz}$. Such disturbance is a relatively common occurrence in road surveys, affected by several sources of cultural noise, e.g., the action induced by towing vehicles.

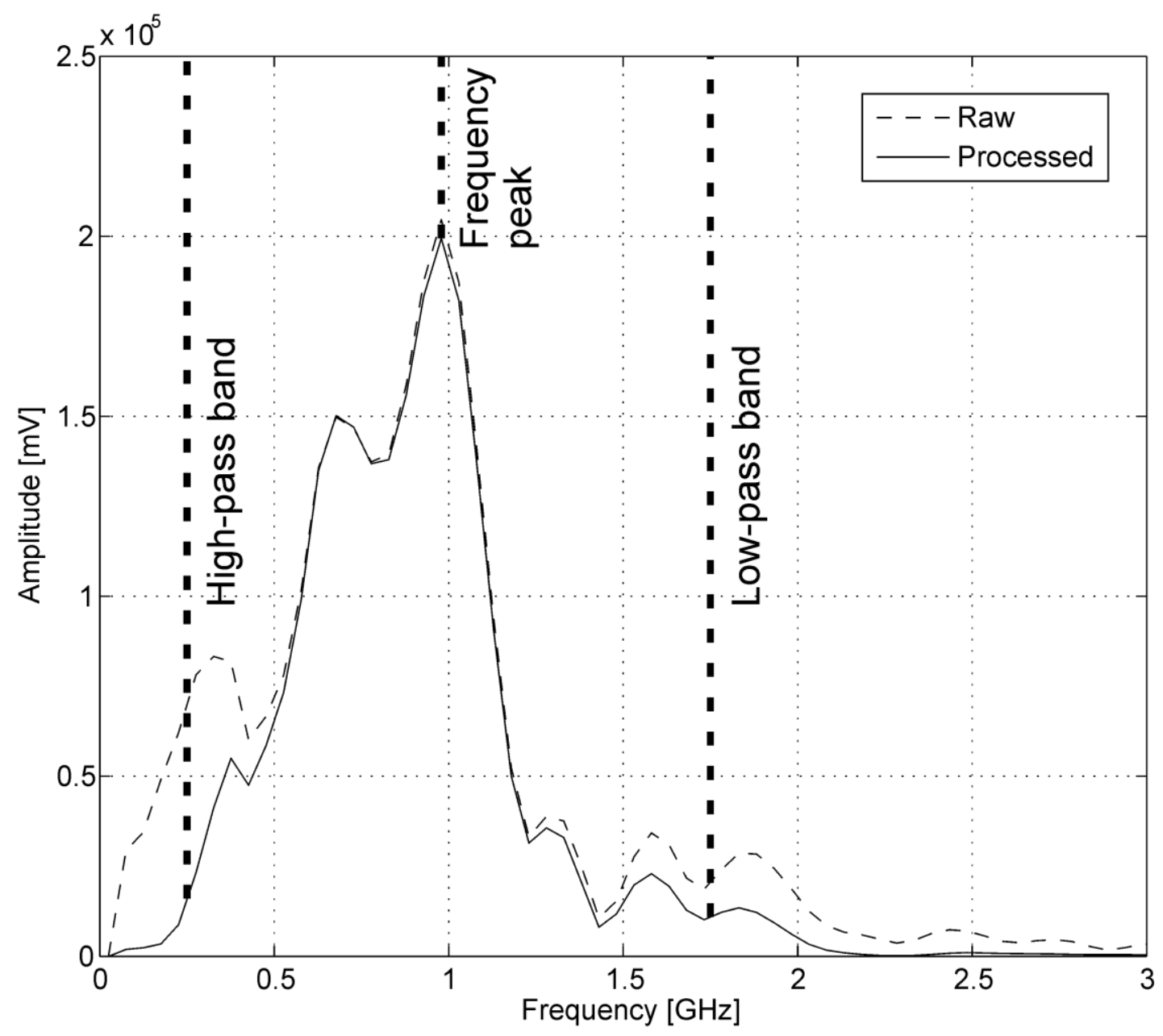


Fig. 5. Band-pass filtering in the frequency spectrum of a GPR signal collected with an air-launched GPR system.

The dashed line shows the shape of the spectrum after the application of a band-pass filter to the spectral region between $250 \mathrm{MHz}$ and $1750 \mathrm{MHz}$. More specifically, if a raw signal trace in the time domain $y(t)$ and its spectral representation $Y(\omega)$ are accounted for, the band-pass filter $H(\omega)$ operates as follows:

$$
Y^{\prime}(\omega)=Y(\omega) \cdot H(\omega)
$$

with $Y^{\prime}(\omega)$ being the processed data in the frequency domain. Several different types of band-pass filters, affecting the shape of the reconstructed spectrum, can be found in the literature. One of the most common is the Butterworth filter, expressed by (13) as follows:

$$
H(\omega)=\frac{B^{(n)}\left(\frac{\omega}{\omega_{c}}\right)}{B^{(m)}\left(\frac{\omega}{\omega_{c}}\right)}
$$

where $B^{(k)}(\omega)$ is a $k$-order Butterworth polynomial, and $\omega_{c}$ is the cutting-off frequency, which depends on whether a low- or a high-pass filter is considered first. There is no univocal rule for setting the width of the pass band, but it is rather a choice that needs to be undertaken after the observation of the signal spectra. Indeed, the risk of cutting-off frequency regions with likely relevant information, hidden by some noise, is relatively high. An effective and not too impactful practice is to set a pass bandwidth of 1.5 times the central frequency [16]. It is worth noting that the antenna-ground coupling mostly generates a shift of the central frequency towards lower values than the nominal frequency, due to the coupling effects between the antenna and the surface. Thereby, it is highly recommended to check this feature prior to set the pass band centered around the nominal frequency of the antenna, as provided by manufacturer. This issue is particularly relevant in road inspections.

\subsubsection{Time-varying gain}

Due to the dispersive nature of the EM wave and to the geometrical spreading losses, the GPR signal suffers by an attenuation when propagating through a medium. The intensity of such attenuation is related to the electrical conductivity of the passed through medium [4]. Mostly in case of high conductivity materials, such 
as clayey soils, deeper targets can be hardly detected. It can be worth to compensate the loss suffered by the signal when applying a time-varying gain to each A-scan [16, 28]. Time gains actually alter the radar signal, and therefore have to be applied with care, in order to avoid the addiction of artifacts. This processing procedure is most likely to work effectively when the data are clear of noise, or after a well-performed decluttering. Indeed, if a gain function is applied to a noisy signal, the late-arrival noise components will be enhanced, thereby inducing potentially incorrect data interpretation.

The general form of a time-varying gain function can be expressed as follows:

$$
y^{\prime}(n)=y(n) \cdot k \cdot n
$$

where $y^{\prime}(n)$ is the $n^{\text {th }}$ sample of the considered trace in the time domain, and $k$ is the gaining function of the sample number $n$ [4]. Several gain functions are commonly employed in GPR data interpretation according to the objective of the data processing. The spherical and exponential (SEC) function operates by compensating the loss of energy caused by geometrical spreading effects [28], with an exponential relationship. On the other hand, the automatic gain compensation (AGC) works by sorting each signal trace in several time windows characterized by different average amplitudes. The compensation applied by the algorithm is a function of the difference between the average amplitude within a time window and the maximum amplitude of the whole trace [34]. In this case, the width of the time windows highly influences the performances of the process. As a rule of thumb, simple constant, linear or exponential gain functions can be applied to the signal, at the discretion of the user. Nevertheless, the choice of the type of gain function should depends on the physical model of the target.

Time-varying gain functions can represent a useful mean for imaging deeper information in road inspections. For instance, they may help in reconstructing the configuration of deeper layers in case of clayey soils. Nevertheless, it is the authors' opinion that the gaining step has to be strictly performed in post-processing, whereas it is preferable to collect the data in their raw nature. Indeed, it can occur to change the gain function afterwards, e.g., in case of information about the subsurface acquired subsequently, or choosing to avoid the application of any gain function, such as in case of noisy records.

\subsubsection{Resolution improvement methods}


Concerning pulsed GPR systems, the smallest dimension of the detectable targets is strictly dependent on the central frequency employed, as well as on the dielectric permittivity of the material that constitutes the target. According to [35], for a typical Ricker wavelet with a central frequency close enough to the bandwidth, the smallest detectable thickness $s_{\min }$ is:

$$
S_{\min }=\frac{\lambda_{0}}{2 \sqrt{\varepsilon_{r, m}}}
$$

with $\lambda_{0}$ and $\varepsilon_{r, m}$ being the wavelength of the EM wave in the air and the dielectric permittivity of the target, respectively. In general, the resolution of the system can be described as the minimal time step $\Delta t$ between two consecutive recognizable echoes, at a fixed frequency bandwidth $B$. Thereby, the system resolution power is characterized by the factor $B \cdot \Delta t$. In case two different targets are too close each other, it is also affected by ringing effects that limit the capability of recognizing such targets, such as in seismic applications [22].

A road pavement can be typically simplified as a non-dispersive ensemble of homogeneous horizontal layers. Usually, the thickness of the first layers, namely, the hot-mix asphalt (HMA) layers, ranges between $1 \mathrm{~cm}$ and $6 \mathrm{~cm}$, thereby falling behind the resolution power of the system. It is therefore frequent that an overlap of the reflections from the top and the bottom interfaces of the HMA layer may occur, due to the central frequency employed. This is typically referred to as 'thin layers problem'. The HMA layer thickness is a key parameter to consider in pavement design processes, and once the pavement is constructed, it covers a primary role in both quality control and quality assurance surveys, within the frame of effective road asset management plans $[9,36-38]$.

One of the most acknowledged processing methods capable of overcoming such an issue is the deconvolution technique, which relies on the assumption that the recorded GPR signal $x(t)$ can be modeled as the convolution between the transmitted wavelet $w(t)$ and the target reflectivity $e(t)[39,40]$, as expressed by (16):

$$
x(t)=w(t) * e(t)
$$

The deconvolution technique consists in isolating the $e(t)$ function by accounting for the reflected signal and retrieving the source wavelet by means of calibration or statistical inferences. Depending on the way $w(t)$ is calculated, deconvolution techniques can be roughly sorted in deterministic and stochastic. 
Deterministic deconvolution is based on an a priori knowledge of $w(t)$, typically assessed by calibration, which in far field conditions is easily achieved by performing GPR tests over a plate of perfect electric conductor (PEC) [41]. In near field conditions, e.g., in case of ground-coupled antenna systems, an accurate determination of the source wavelet is more difficult, thereby making such technique less applicable. The deterministic approach is definitely more straightforward and efficient to undertake under a numerical point of view. Positive outcomes are documented in [42,43] and [44], wherein the authors performed a deconvolution as a simple fraction in the frequency domain and by means of a matricial approach, respectively.

Stochastic methods aim at developing a blind deconvolution in which the only back-reflected GPR signal is known. These methods rely on statistical and numerical processes for defining the transmitted wavelet. Depending on the process, several seismic methods can be listed within the family of stochastic methods applicable to GPR, including propagation deconvolution [45], predictive deconvolution [46], two-sided deconvolution [47], source signature deconvolution [48], Wiener deconvolution [47], deconvolution via sparsity maximization [42], and super- and high-resolution methods [50, 51]. The nature of these methods results in a major computational complexity, which in turn allows increasing the resolution without calibrating the system.

Nevertheless, deconvolution is by definition a solution to an ill-posed problem [52], characterized by important approximations, thereby involving an estimation of a solution rather than an exact solution. Moreover, notwithstanding the acknowledged outcomes achieved in the seismic discipline, the effectiveness of such a technique in GPR applications, wherein the considered wavelet is generally mixed-phase and nonstationary, has been frequently a subject matter of discussion $[16,17]$.

\subsection{B-scan processing}

\subsubsection{Background removal}

If an ensemble of traces is considered, the clutter can be reduced by subtracting from each A-scan the average value of the amplitude related to a singular sample, calculated over the whole set of A-scans. The procedure can be explained as follows [4]: 


$$
y^{\prime}(n)=y(n)-\frac{1}{K} \sum_{k=1}^{K} y_{k}(n)
$$

where $y^{\prime}(n)$ and $y(n)$ are the processed and raw signal traces, respectively, with $n$ being the number of the sample and $k$ being the number of the trace within the selected set of A-scans. In Fig. 6, a B-scan before (a) and after (b) the application of the background removal filter is reported.

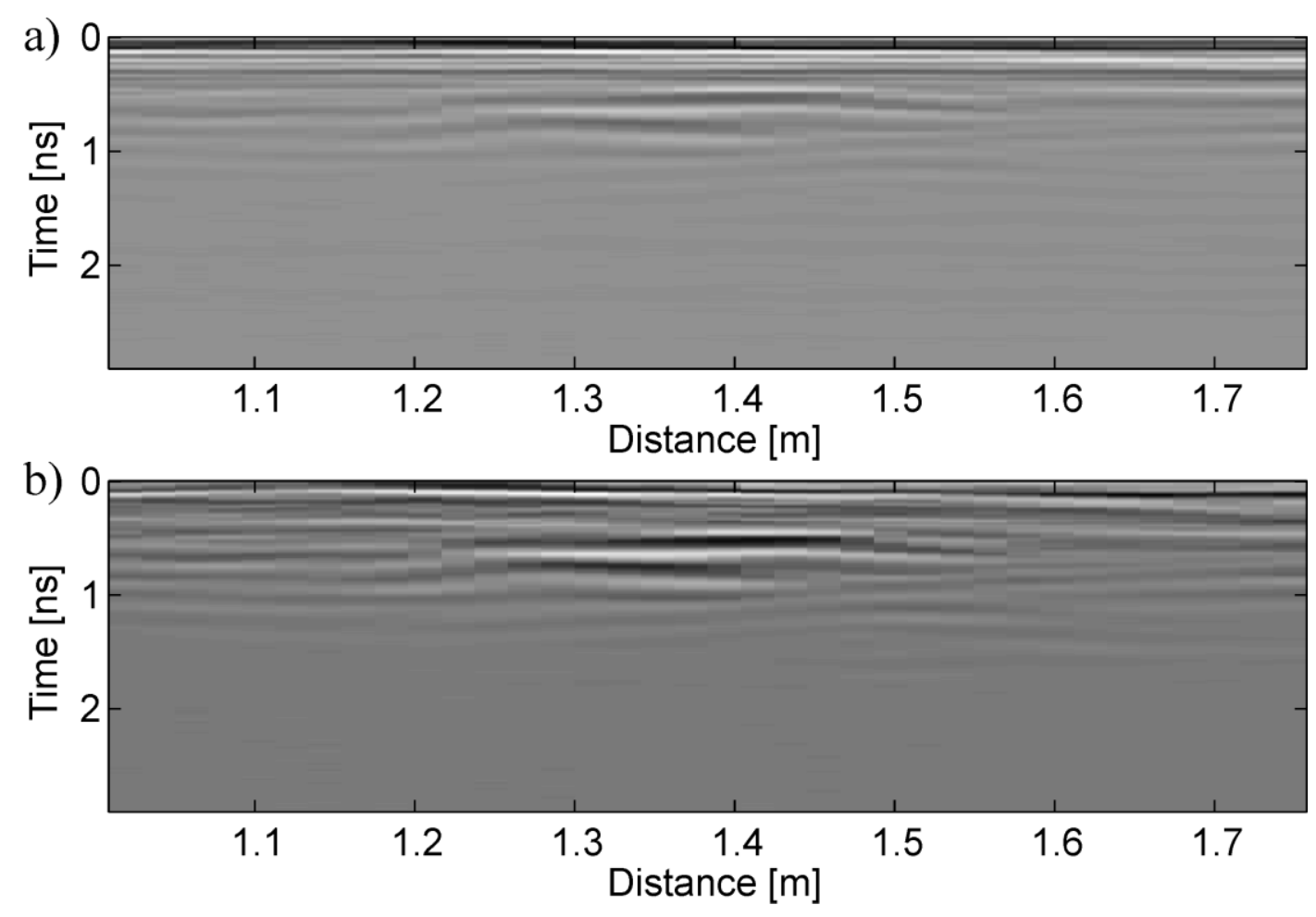

Fig. 6. Application of the background removal filter: B-scan before (a) and after (b) applying the background removal.

It is evident how the removal of the background noise enhances the subsurface reflections. In particular, a region interested by unevenness in the pavement configuration is clearly recognizable within approximatively $8 \mathrm{~m}$ and $13 \mathrm{~m}$. On the other hand, it is worth noting that flat layers, such as the air-pavement interface, have been totally removed. This is a major assumption when dealing with road inspections, since the pavement layers are supposed to be horizontal in respect to the survey track. In this sense, the background removal is not useful in case the analysis of the layer thicknesses is set as objective. Conversely, if the objective of the processing is to highlight any deviations within the pavement configuration, it can represent an effective technique. Lastly, it is worth mentioning that this processing technique holds the potential of introducing artifacts in presence of homogeneous non-reflecting areas [28]. In general, an 
experienced operator is required when applying a background removal filter to a dataset in order to avoid misinterpretations.

\subsubsection{Velocity analysis}

Road inspections usually require a proper time-depth conversion of the received signal, such as in most GPR applications. Commercial processing software usually achieve this automatically, by employing a constant value of velocity of the propagating wave. Also neglecting the possible inhomogeneous distribution of water content and air-filled voids, which highly affect the wave velocity, this procedure will inevitably lead to a wrong conversion in the case of roads. Indeed, the assumption of a constant value of velocity is a simplifying hypothesis that leads to a systematic error due to the multi-layered nature of road pavements, which are constituted of materials with different permittivity values. In addition, the relative real part of the dielectric permittivity $\varepsilon_{r}$ ' is directly related to the propagation velocity of the EM wave in the medium $(v)$ and in the vacuum $\left(c_{0}\right)$ as explained in (18) [53]:

$$
v=\frac{c_{0}}{\sqrt{\varepsilon_{r^{\prime}}}}
$$

An accurate estimation of the subsurface wave propagation velocity within the media can be achieved through different methods. The first one, which lies beyond the context of signal processing, is the traditional ground truthing. This is mostly performed by coring the pavement along the survey track in order to visually measure the thicknesses of the layers. If a suitable number of cores is gathered, it is possible to interpolate, mostly linearly, the depth of the interfaces and reconstructing the configuration of the pavement structure along the whole length of the road inspected. Notwithstanding the high accuracy of the measurement in the closeness of the core, this method has many drawbacks, amongst which we can cite the invasiveness, the high costs, and the low representativeness of the real conditions over the distance between two consecutive cores, especially in case the number of cores is limited. The main methods relevant to the GPR practices are the "common midpoint" (CMP), and the "hyperbolic velocity analysis". More than a processing technique, the CMP technique is a survey methodology based on the use of a bi-static GPR system collecting data at different configurations. After each data collection, the emitting and receiving antennas are moved aside of a same distance each other, such that the midpoint between is kept fixed (Fig. 7). In case of a horizontal target reflecting the GPR signal in the subsurface, located at depth $z$ and constant for all the test configurations, the 
two-travel time $t$ of its reflection will increase as the offset between the transmitter and the receiver $(x)$ grows. The propagation velocity $v$ can be defined by geometrical assumptions [54]:

$$
\begin{aligned}
& t^{2}=\frac{x^{2}}{v^{2}}+\frac{4 z^{2}}{v^{2}} \\
& v=\frac{2 \sqrt{z^{2}+\left(\frac{x}{2}\right)^{2}}}{t}
\end{aligned}
$$

As road pavements are mostly multi-layered targets, the velocity has to be calibrated with regard to the $n^{\text {th }}$ layer. (18) can be so extended as [55]:

$$
v_{n}=\frac{\sqrt{t_{n} v_{n}^{2}-t_{n-1} v_{n-1}^{2}}}{t_{n}-t_{n-1}}
$$

Simple corrections to (20) and (21) allows extending the results to the case of dipping targets:

$$
v_{n}=\frac{\sqrt{t_{n} v_{n}^{2} \cos ^{2} \varphi-t_{n-1} v_{n-1}^{2} \cos ^{2} \varphi}}{t_{n}-t_{n-1}}
$$

with $\varphi$ being the slope angle of the dipping target with respect to the surface.

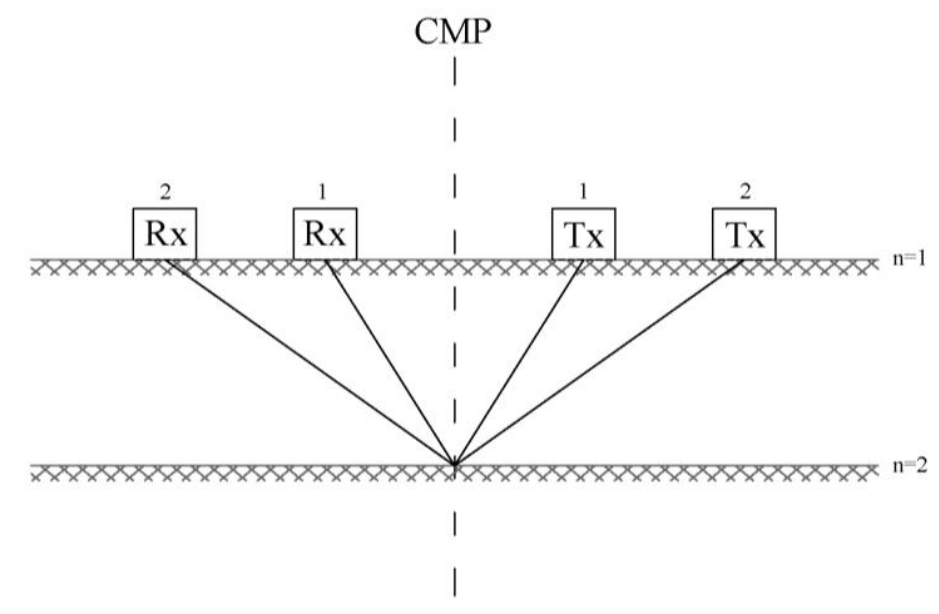

Fig. 7. Configuration of antennas in a CMP acquisition.

On the contrary, the hyperbolic velocity analysis can be applied only to sections with a clear hyperbolic diffraction, but holds the advantage of being applicable also with a mono-static GPR device or with a GPR system which does not allow to move transmitter and receiver aside. If the B-scan of a section containing the hyperbola is taken into account, a hyperbolic curve can be hypothesized by arbitrary choosing a value of wave velocity $v$ ' to be used in (19). By iteratively using new values of $v$ ' and by comparing the shape of the 
expressed curve with the hyperbola generated by the reflector, it is possible to set the value $v^{*}$ whereby the two curves match as well as the propagation velocity of the wave through the medium.

By coupling more than one antenna transversally to the scan direction, it is possible to achieve a real-time multi-offset analysis. The extended CMP method (XCMP method), proposed in [56, 57], exploits the use of an air-coupled antenna array equipping a stepped-frequency GPR with the aim of continuously evaluating the thickness of the HMA layer in asphalt pavements.

\section{CONCLUSIONS}

This paper aims at discussing the main GPR processing techniques applied in road inspections. First, an introduction of the GPR practices in road engineering is given. Then, an overview of the working principles of a GPR system in its most common configurations is provided. The core of the paper focuses on the processing methodologies mostly employed in the interpretation of data collected in road pavements, which constitute part of the acknowledged techniques used in the GPR practice within other disciplines and applications. A thorough work on the most advanced GPR processing techniques embracing several scientific disciplines is provided in [4]. It is worthwhile noting that in most cases, the more complex the processing is, the higher is the risk of introducing artefacts in the data, which can lead to wrong interpretations. Furthermore, it is worthy to be mentioned how each processing technique is suitable for a specific purpose. It is then crucial to set the objectives prior to process the data, in order to choose the relevant processing schemes, such that it would be more likely to avoid distortion and over-processing of the data and, mostly, waste of time. It is also useful to remember that processing has a cost, which mainly consists in the use of human resources. Even more in the case of road inspections, characterized by a huge amount of data, it is mandatory to invest time and efforts in analyzing the data collected before the processing, to check the main requirements and deliverables, and the most effective strategy for reaching the goal of the survey. Thereby, it is worth mentioning how the quality of the dataset influences the intensity of the processing needed for increasing its readability. At the same time, the level of noise and the setting of the proper frequency in line with the nature of the target may also represent a limit to the performance of the 
processing phase. Accordingly, the proper performance of a GPR survey on the site is the fundamental and most important factor to pursue, in order to emphasize the effectiveness of the data processing.

\section{ACKNOWLEDGEMENT}

This work has benefited from the network activities carried out within the EU funded COST Action TU1208 “Civil Engineering Applications of Ground Penetrating Radar".

\section{REFERENCES}

1. S. Tighe, N. Li, L.C. Falls, R. Haas, Incorporating road safety into pavement management, Transp. Res. Rec. 1699 (2000) 1-10.

2. C. Maierhofer, Planning a non-destructive test programme for reinforced concrete structures, NonDestructive Evaluation of Reinforced Concrete Structures, Woodhead, (2010) 3-13,

3. A. Benedetto, F. D'Amico, F. Tosti, Improving safety of runway overrun through the correct numerical evaluation of rutting in Cleared and Graded Areas, Saf. Sci. 62 (2014) 326-338.

4. D.J. Daniels, Ground Penetrating Radar, The Institution of Electrical Engineers, London, 2004.

5. E.C: Slob, M. Sato, G. Olhoeft, Surface and borehole ground-penetrating-radar developments, Geophysics 75 (5) (2010) 75A103-75A120.

6. R. More, Ground Penetrating Radar for evaluating subsurface conditions for transportation facilities, Synthesis of Highway Practice 255, National Cooperative Highway Research Program, Transportation Research Board, 1998

7. I.L. Al-Qadi, S. Lahouar, Use of GPR for thickness measurement and quality control of flexible pavements, in: Proceedings of Asphalt Paving Technology: Association of Asphalt Paving Technologists, (2004) 501-528.

8. T. Scullion, L. Lau, Y. Chen, Implementation of the Texas ground penetrating radar system,” Tech. Rep. 1233-1, Texas Transportation Institute, College Station, Tex, USA, 1992

9. T. Scullion, T. Saarenketo, Road evaluation with ground penetrating radar, J. Appl. Geophys. 43 (2000) $119-138$. 
10. D. Huston, N. Pelczarski K. Maser, Damage detection in roadways with Ground Penetrating Radar, in: Proceedings 8th International Conference on Ground Penetrating Radar, Gold Coasts, Australia, May 2000.

11. A. Benedetto, G. Manacorda, A. Simi, F. Tosti, Novel perspectives in bridges inspection using GPR, Nondestruct. Test. Eva. 27 (3) (2012) 239-251.

12. A. Benedetto, F. Tosti, Inferring bearing ratio of unbound materials from dielectric properties using GPR: The case of runaway safety areas, in: Proceedings of the 2013 Airfield and Highway Pavement Conference: Sustainable and Efficient Pavements, 1336-1347, June 2013.

13. F. Tosti, S. Adabi, L. Pajewski, G. Schettini, A. Benedetto, Large-scale analysis of dielectric and mechanical properties of pavement using GPR and LFWD, in: Proceedings of the 15th International Conference on Ground Penetrating Radar, 868-873, June-July 2014.

14. A. Benedetto, F. Tosti, L. Pajewski, F. D'Amico, W. Kusayanagi, FDTD simulation of the GPR signal for effective inspection of pavement damages, in: Proceedings of the 15th International Conference on Ground Penetrating Radar, 513-518, June-July 2014.

15. ASTM D6087-08, Standard test method for evaluating asphalt-covered concrete bridge decks using Ground Penetrating Radar, ASTM International, West Conshohocken, PA, 2008.

16. H. Jol, Ground Penetrating Radar, Elsevier, 2009.

17. A. Benedetto, L. Pajewski, Civil engineering applications of Ground Penetrating Radar, Springer, 2015.

18. M. Gagarin, J. Mekemson, Step-frequency ground-penetrating-radar array calibration requirements to estimate dielectric properties of pavements, 14 (2) (2016) 105-110.

19. S. Lahouar, I.L. Al-Qadi, A. Loulizi, T.M. Clark D.T. Lee, Approach to determining in situ dielectric constant of pavements: development and implementation at interstate 81 in Virginia, Transp. Res. Rec., 1806 (1) (2002) 81-87.

20. A. Loizos, C. Plati, Accuracy of pavement thicknesses estimation using different ground penetrating radar analysis approaches, NDT and E Int. 40 (2) (2007) 147-157.

21. O. Yilmaz, Seismic data processing (investigations in geophysics, vol. 2), Society of Exploration Geohysicists, Tulsa, 1987. 
22. O. Yilmaz, Seismic data analysis (investigations in geophysics, no. 10), Society of Exploration Geophysicists, 2001.

23. G.R. Olhoeft, Document maximizing the information return from ground penetrating radar, J. Appl. Geophys. 43 (2-4) (2000) 175-187.

24. D.C. Nobes, Geophysical surveys of burial sites: a case study of the Oaro urupa, Geophysics 64 (2) (1999) 357-367.

25. R. Yelf, D. Yelf; Where is true time zero?, Electromag. Phenom. 7, 1 (18) (2006) 159-163.

26. E.R. Dougherty, Optimal mean-absolute-error filtering of gray-scale signals by the morphological hit-ormiss transform, J. Math. Imaging Vis. 4 (3) (1994) 255-271.

27. K. Gerlitz, M.D. Knoll, G.M. Cross, R.D. Luzitano, R. Knight, Processing ground penetrating radar data to improve resolution of near-surface targets, in: Proceedings of the Symposium on the Application of Geophysics to Engineering and Environmental Problems, San Diego, California, April 1993.

28. A.P. Annan, Practical processing of GPR data, Sensors and Software Inc., 1999.

29. N. Economou, A. Vafidis, Spectral balancing GPR data using time-variant bandwidth in the t-f domain, Geophysics 75 (3) (2010) J19-J27.

30. J. Sun, R.A. Young, Recognizing surface scattering in ground-penetrating radar data, Geophysics 60 (5) (1995) 1378-1385.

31. M. Bano, F. Pivot, J.M. Methelot, Modelling and filtering of surface scattering in ground-penetrating radar waves, Wiley Online Library 17 (6) (1999) 215-222.

32. L. Nuzzo, Coherent noise attenuation in GPR data linear and parabolic Radon Transform techniques, Ann. Geophys.-Italy 46 (3) (2003) 533-547.

33. S. Malagodi, L. Orlando, S. Piro, F. Rosso, Location of archaeological structures using the GPR method:three-dimensional data acquisition and radar signal processing. Archaeol. Prospect. 3 (1) (1996) $13-23$.

34. H. Horstmeyer, M. Gurtner, F. Büker, A.G. Green,. Processing 2-D and 3-D georadar data: some special requirements, in: Proceedings 2nd Meeting Environmental and Engineering Geophysics Society (EEGSES), Nantes, France, 264-267, 1996. 
35. U. Spagnolini, Permittivity measurements of multilayered media with monostatic pulse radar, IEEE T. Geosci. Remote 35 (2) (1997) 454-463.

36. Y.H. Huang, Pavement analysis and design, Pearson Prentice Hall, 1993.

37. T. Scullion, T. Saarenkeeto, Using suction and dielectric measurements as performance indicators for aggregate base materials, Transp. Res. Rec. 1577 (1997) 37-44,.

38. P. Shangguan, I.L. Al-Qadi, A. Coenen, S. Zhao, Algorithm development for the application of groundpenetrating radar on asphalt pavement compaction monitoring, Int. J. Pav. Eng. 17 (3) (2016) 189-200.

39. S.M. Riad, The deconvolution problem: an overview, IEEE T. Geosci. Remote 74 (1) (1986) 82-85.

40. G. Turner, Propagation deconvolution, in: Proceedings of the 4th International Conference on Ground Penetrating Radar, 85-93, June 1992.

41. K. Chahine, V. Baltazart, Y. Wang, X. Dérobert, Blind deconvolution via sparsity maximization applied to GPR data, Eur. J. Environ. Civil Eng. 15 (4) (2011) 575-586.

42. J. Xia, E. K. Franseen, R. D. Miller, T. V. Weis, A. P. Byrnes, Improving ground-penetrating radar data in sedimentary rocks using deterministic deconvolution, J. Appl. Geophys. 54 (2003) 15-33.

43. L. Wei, H.M. Lei, P.W. Que, Sparsity enhancement for blind deconvolution of ultrasonic signals in nondestructive testi application, Rev. Sci. Instrum. (79) 1, (2008) 014901-1-014901-6.

44. S. Zhao, P. Shangguan, I. L. Al-Qadi, Application of regularized deconvolution technique for predicting pavement think layer thickness from ground penetrating radar data, NDT\&E Int. 73 (2015) 1-7.

45. G. Turner, Subsurface radar propagation deconvolution, Geophysics 9 (22) (1994) 215-223.

46. J.P. Todoeschuck, P.T. LaFlèche, O.G. Jensen, A.S. Judge, J.A. Pilon, Deconvolution of ground probing radar data: in ground penetrating radar, ed. J. Pilon, Geological Survey of Canada, paper 90-4, 227-230, 1992.

47. F.M. Gottsche, C. Stolte, K.P. Nick, Two-sided deconvolution: a method to improve the temporal resolution in radar data, in: Proceedings of the 56th meeting of European Association of Exploration Geophysicists, June 1994.

48. F.A. Neves, J.A. Miller, M.S. Roulston, Source signature deconvolution of ground penetrating radar data, in: Proceedings of the 6th International Conference on Ground Penetrating Radar, 573-578, September-October 1994. 
49. M.L. Moran, R.J. Greenfield, S.A. Arcone, A.J. Delaney, Delineation of a complexity dipping temperate glacier bed using short-pulse radar arrays, J. Glaciol. 46 (2000) 274-286.

50. J. Li, R. Wu, An efficient algorithm for time delay estimation, IEEE T. Signal Proces., 46 (8), (1998) 2231-2235.

51. C. Le Bastard, V. Baltazart, X. Dérobert, Y. Wang, Support vector regression method applied to thin pavement thickness estimation by GPR, in: Proceedings of the 14th International Conference on Ground Penetrating Radar, 349-353, June 2012.

52. A. N. Tykhonv, V.Y. Arsenin, Solution of Ill-posed Problems, Math.Comput. 32 (144) (1978) 13201322.

53. C. Sperl, Determination of spatial and temporal variation of the soil water content in an agro-ecosystem with ground-penetrating radar (In German). Ph.D. Thesis Technische Universitat Munchen, Munich, Germany, 1996.

54. T. Tillard, J.C. Dubois, Influence and lithology on radar echoes: analysis with respect to electromagnetic parameters and rock anisotropy, in: Proceedings of the 4th International Conference on Ground Penetrating Radar, 95-102, June 1992.

55. C. H. Dix, Seismic velocities from surface measurements, Geophysics 20 (1955) 68-86.

56. Z. Leng, I.L. Al-Qadi, An innovative method for measuring pavement dielectric constant using the extended CMP method with two air-coupled GPR systems, NDT\&E Int. 66 (2014) 90-98.

57. S. Zhao, I.L. Al-Qadi, Development of an analytic approach utilizing the extended common midpoint method to estimate asphalt pavement thickness with 3-D ground-penetrating radar, NDT\&E Int. 78 (2016) 29-36. 